Viviane S. Teixeira*, Vera Labitzky, Udo Schumacher and Wolfgang Krautschneider

\title{
Use of Electrical Impedance Spectroscopy to Distinguish Cancer from Normal Tissues with a Four Electrode Terminal Setup
}

\begin{abstract}
Cancer and normal tissues are visually different from each other, especially so in more advanced cancer stages. More important, they are not only visually contrasting, but if an electric field is applied to both tissue types and the frequency is varied in a wide range, it will be seen that the two tissue types in general have a spectral response divergent from each other and this has to do with the characteristics of cancer tissues in contrast to normal ones. In this work, Electrical Impedance Spectroscopy is applied to try to distinguish cancer from healthy tissues by means of their impedance spectrum using a four-electrode-terminal setup. The use of the fourterminal-setup setup is important to circumvent the impact of electrode polarization at frequencies below $1 \mathrm{kHz}$.
\end{abstract}

Keywords: Cancer cells, electrical impedance spectroscopy, four electrode terminal setup, malignant tumors, normal cells, normal tissues, impedance spectrum.

https://doi.org/10.1515/cdbme-2020-3088

\section{Introduction}

Electrical impedance spectroscopy (EIS) is a technique that can possibly be used to distinguish cancer from normal tissues based on the measurement of the electric properties of tissues when submitted to frequency variable electric fields [1-8]. It consists on the application of a small amplitude voltage or current signal and the measurement of the biological tissue response [1]. It is label-free in the sense that no staining is needed and it also does not modify the tissue structure. Very small voltages and currents should be applied in order not to

*Viviane S. Teixeira: Institute for Integrated Circuits, Hamburg University of Technology, Eissendorfer Str. 38, 21073 Hamburg, Germany, viviane.silva.teixeira@tuhh.de.

Vera Labitzky, Udo Schumacher: Institute of Anatomy and Experimentally Morphology, University Medical Center HamburgEppendorf (UKE), Hamburg, Germany.

Wolfgang Krautschneider: Institute for Integrated Circuits, Hamburg University of Technology, Hamburg, Germany. degrade tissue vitality. It is already used in several fields and is currently under development to distinguish cancer cells and tissues from normal ones [1-9].

The interesting aspect about applying EIS to cancer tissues is that they show a spectral response quite different from their normal counterparts and this has all to do with the effects of the cancer growing within a normal tissue environment. Also, important information can be derived from their spectral profile. Cancers in general have larger water and salt content when compared to normal organs, caused by leaky blood vessels and the lack of a properly working lymphatic system [10-13]. This imbalance in fluid transport leads to the accumulation of interstitial fluid, liquids and salts inside the tumour. For EIS, higher water and salt content translates in higher tissue conductivity, and consequently, lower impedance.

\section{Experimental Procedure}

The severe combined immunodeficient (SCID) mouse was purchased from Charles River. The autosomal recessive scid mutation leads to the absence of functional $\mathrm{B}$ and $\mathrm{T}$ lymphocytes, therefore the human tumour cells can be xenografted onto mice of this strain [14].

A total of 1 million human colon carcinoma cells HT29 (ATCC) were subcutaneously injected into SCID mouse in the area of the right scapula. The animal was kept in an individual ventilated cage under pathogen-free conditions, fed with sterile standard food and water ad libitum, and was regularly monitored concerning subcutaneous the tumour growth. When the tumour reached $1 \mathrm{~cm}^{3}$, the animal was sacrificed, the tumour and the organs excised and their impedance measured ex-vivo. Measurements were performed immediately after sacrifice. Organs and primary tumour were placed in phosphate buffer saline (PBS) for the measurements. An array of four needle electrodes was used to measure the impedance and provide a four-terminal stimulation. The needles used had a total length of $12 \mathrm{~mm}$ and a diameter of $0.4 \mathrm{~mm}$. 
The potentiostat Gamry Interface 1000 was used to perform all experiments. A $14 \mathrm{mV}$ amplitude voltage signal was applied and the frequency was varied from $1 \mathrm{MHz}$ up to $100 \mathrm{mHz}$ in steps of ten points per decade.

All results were processed and plotted in MATLAB.

\section{Results}

\subsection{Spectrum of Mouse Normal Organs}

In the brain, both right and left hemispheres were measured in longitudinal and transversal direction. The average of the impedance magnitude and phase for both hemispheres and directions is also shown. Some differences in the measured impedance was observed from longitudinal to transversal directions showing the anisotropy of the brain, while right and left hemispheres did not show significant differences from each other. This anisotropy in the directions is common in biological tissues and is due to the different tissue arrangement from one direction to the other.

In mouse kidneys, the degree of anisotropy observed experimentally between transversal and longitudinal directions was larger than for the brain (see Figure 2). Furthermore, the right kidney showed a lower impedance than the left one. One possible explanation can be that one kidney (right) was full of liquids with salts which decreased its impedance.
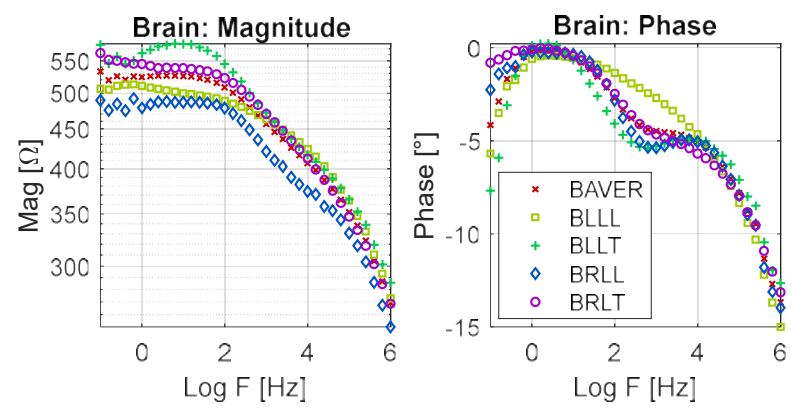

Figure 1: SCID mouse brain impedance magnitude and phase, measured in both hemispheres (left and right) and in longitudinal and transversal directions. Legend: BAVER (brain average), BLLL (brain left hemisphere longitudinal), BLLT (brain left hemisphere transversal), BRLL (brain right hemisphere longitudinal) and BRLT (brain right hemisphere transversal).

The mouse liver was measured in longitudinal and transversal directions (Figure 4). Figure 5 show the results from the impedance measurements from the thigh muscle of one leg of the mouse. The muscle in general has a high salt and water content, therefore it shows a smaller impedance when compared to other organs.
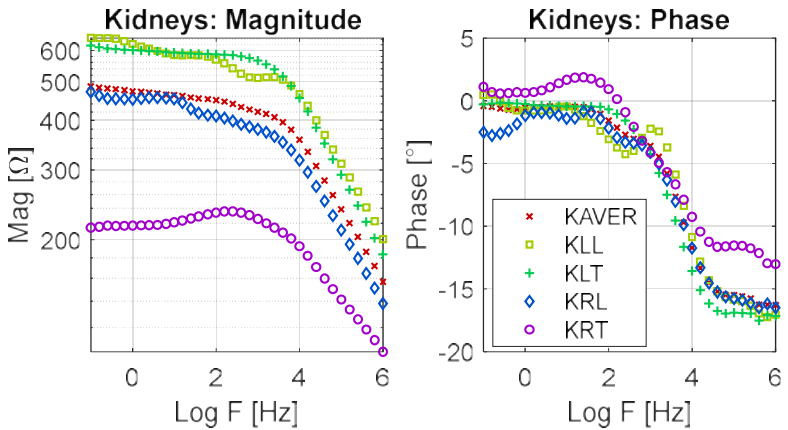

Figure 2: SCID mouse kidney impedance measured in right and left side kidneys, in longitudinal and transversal directions. The average (aver) is plotted in red. Legend: KAVER (kidneys average), KLL (kidney left longitudinal), KLT (kidney left transversal), KRL (kidney right longitudinal) and KRT (kidney right transversal).
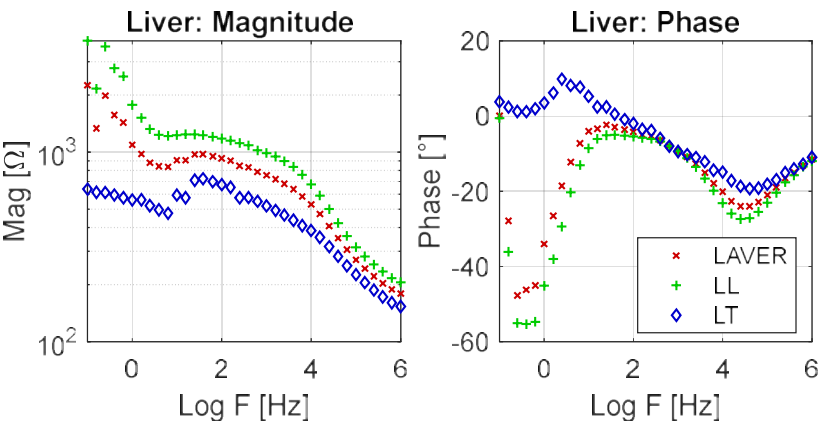

Figure 4: Mouse liver impedance measured in longitudinal and transversal directions. Legend: LAVER (liver average), LL (liver longitudinal) and LT (liver transversal).
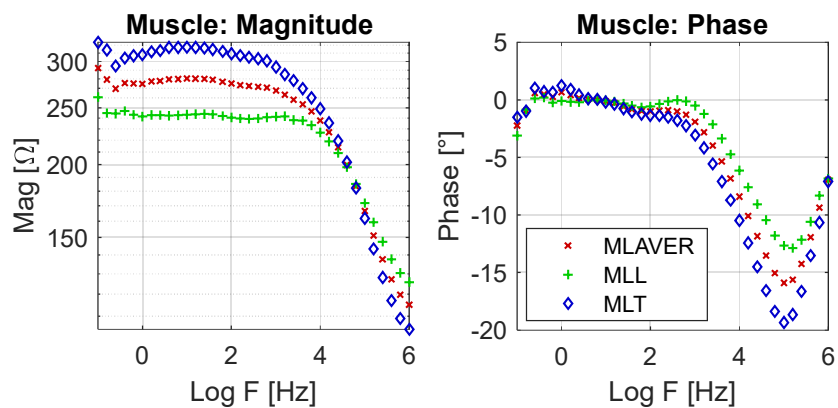

Figure 5: Mouse thigh muscle leg impedance measured in longitudinal and transversal directions. Legend entries: MLAVER (muscle leg average), MLL (muscle leg longitudinal), MLT (muscle leg transversal).

The mouse heart was measured in both longitudinal and transversal directions, which also showed a significant anisotropy in the impedance measured between both directions (Figure 6). The lungs had a large impedance presumably due to their high air content when compared to other organs. 

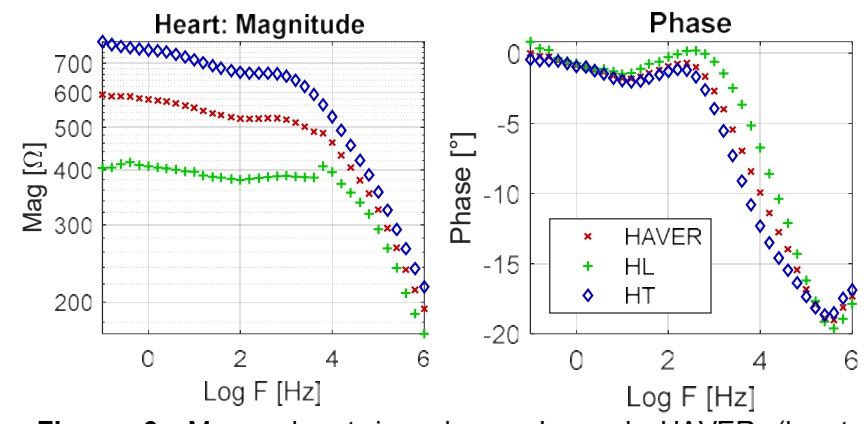

Figure 6: Mouse heart impedance. Legend: HAVER (heart average), $\mathrm{HL}$ (heart longitudinal) and $\mathrm{HT}$ (heart transversal).
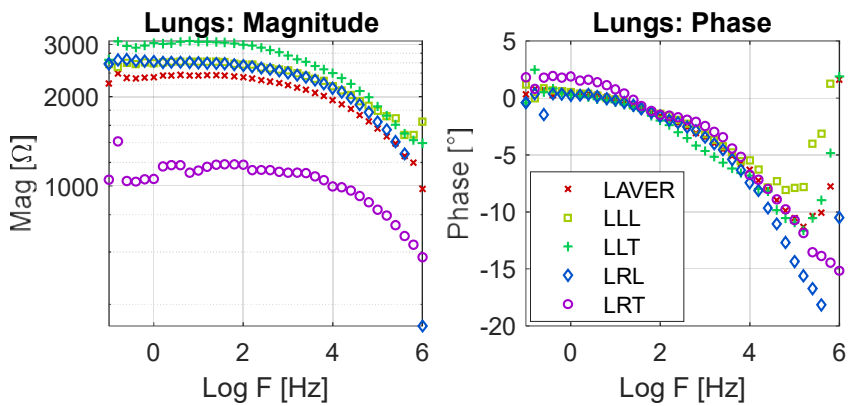

Figure 7: Lungs impedance. Legend: LLAVER (lungs average), LLL (left lung longitudinal), LLT (left lung transversal), LRL (lung right longitudinal) and LRT (lung right transversal).

\subsection{Tumor vs. Normal Tissues Spectrum}

As cancer can form distant metastases in almost any organ it is of interest to plot impedance magnitude and phase of the primary tumour nodule with other organs (Figures 8-10). The primary tumour nodules had the lowest impedance from all measured organs or equivalently the highest conductivity. In principle, the same results were observed in another study with two different tumour types and mice implanted with UT-SCC5 (tongue squamous cell carcinoma) and UM-SCC-10A (laryngeal squamous cell carcinoma), using a two-electrodeterminal setup [1]. An explanation for such behavior is the accumulation of interstitial fluid inside the tumours due to leaky blood and mal-functioning lymphatic vessels [10-13]. Higher water and salt content, which are major ingredients of the interstitial fluid, will cause a decrease in the measured impedance magnitude of the tumour when compared to normal organs. Equivalently, as the conductivity is derived from the real part of the impedance magnitude, a lower magnitude will translate into a higher conductivity.

Interesting to note is that the impedance values correlate closely to organ structure, water and salt content (Figures 8 10). Organs with high water and salt content, e.g. muscle, showed a lower impedance magnitude or equivalently higher conductivity. Conversely, organs e.g. lungs with low liquid content presented the lowest conductivity, equivalent the highest impedance of all measured organs.

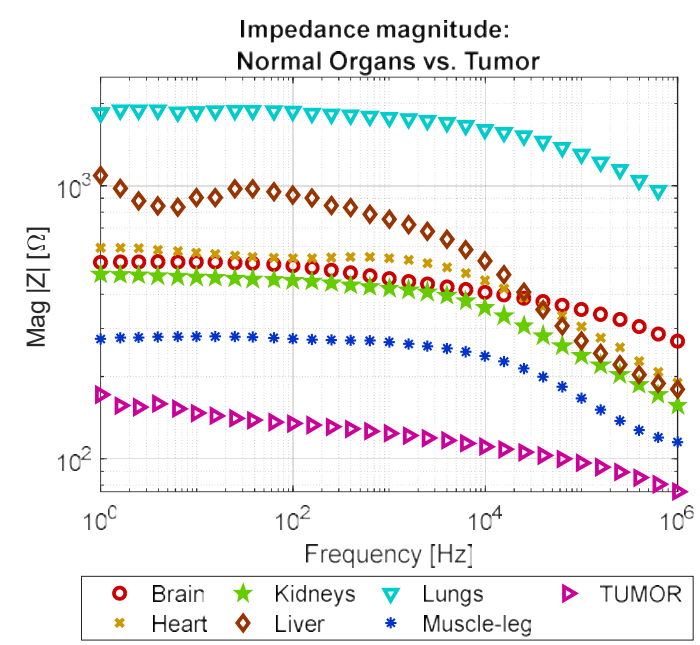

Figure 8: Impedance magnitude of mouse organs plotted together with tumour. All values are averages.

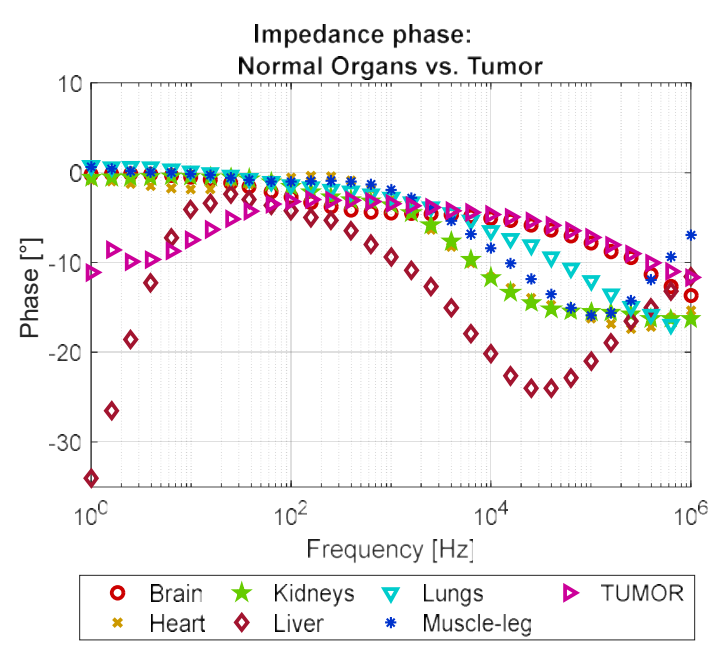

Figure 9: Impedance phase of mouse organs and tumor.

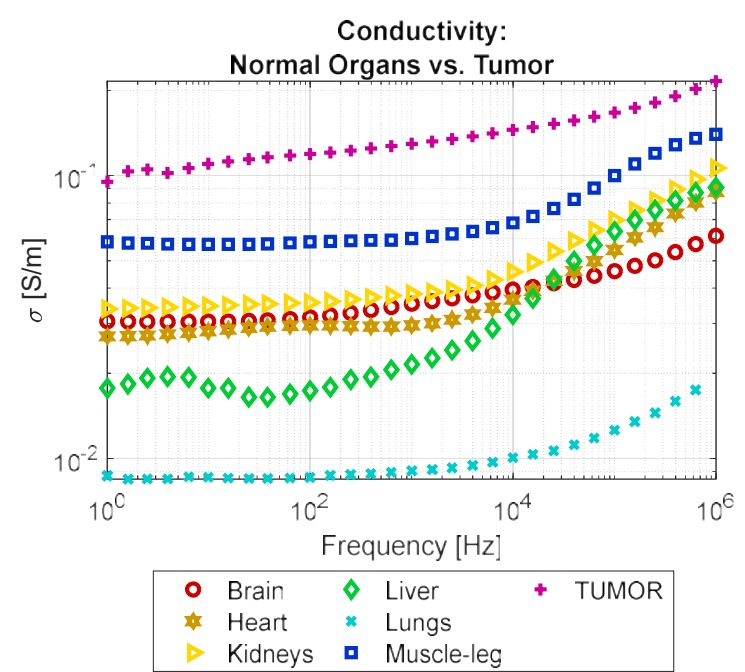

Figure 10: Conductivity of mouse normal organs and primary tumour nodule. Tumours showed the highest conductivity among all measured organs. 
Table 1: Electrical Parameters Mouse Normal Tissues \& Primary Tumor

\begin{tabular}{llll|lll}
\hline Organ & \multicolumn{3}{c|}{ Mag $|\mathbf{Z}| \mathbf{\Omega}]$} & \multicolumn{3}{c}{ Phase $|\mathbf{Z}|\left[^{\circ}\right]$} \\
\hline & $\mathbf{1 k H z}$ & $\mathbf{1 0 k H z}$ & $\mathbf{1 0 0 k H z}$ & $\mathbf{1 k H z}$ & $\mathbf{1 0 k H z}$ & $\mathbf{1 0 0 k H z}$ \\
Lung & 1868 & 1773 & 1301 & 0 & -3 & -13 \\
Liver & 905 & 754 & 270 & -4 & -9 & -21 \\
Heart & 568 & 543 & 305 & -2 & -3 & -16 \\
Brain & 525 & 456 & 352 & 0 & -4 & -8 \\
Kidney & 462 & 420 & 239 & 0 & -3 & -15 \\
Muscle & 280 & 267 & 166 & 0 & -2 & -16 \\
Tumor & 147 & 124 & 97 & -7 & -3 & -7 \\
\hline
\end{tabular}

Table 1 cont.

\begin{tabular}{llll}
\hline Organ & \multicolumn{3}{c}{$\boldsymbol{\sigma}[\mathbf{m S} / \mathbf{m}]$} \\
\hline Lung & $\mathbf{1 k H z}$ & $\mathbf{1 0 k H z}$ & $\mathbf{1 0 0 k H z}$ \\
Liver & 18 & 9 & 12 \\
Heart & 28 & 21 & 64 \\
Brain & 30 & 35 & 55 \\
Kidney & 35 & 38 & 46 \\
Muscle & 57 & 60 & 100 \\
Tumor & 110 & 130 & 170 \\
\hline
\end{tabular}

Table 1 shows that the conductivity of the tumour is more than ten times higher than the lung conductivity, and approximately twice higher as the conductivity of the mouse thigh muscle, which is also an organ rich in water and salt content. The brain, the kidneys and the heart have similar values, with small differences while liver showed also small conductivity values, therefore higher impedance values.

\section{Conclusion}

The results of this experiment showed that the distinction between tumour and normal tissues may be possible by means of Electrical Impedance Spectroscopy. In a previous experiment [1], we obtained similar results using a twoelectrode-terminal setup and three other mice, one healthy and two implanted with UT-SCC-5 cell line (tongue squamous cell carcinoma) and UM-SCC-10A (laryngeal squamous cell carcinoma). In that case the two tumours also showed the lowest impedance among all compared organs. In a large set of experiments recently performed where we measured the same tumour type (human prostate cancer PC-3 cells) in five different mice, we observed the same tendency of the tumours to have either the smallest impedance or to be amongst the smallest together with mouse thigh muscle legs (own unpublished results). This bring us to expect that there is a tendency of tumours to have smaller impedance than healthy organs due to their internal composition: high water and salt content, loose connection between adjacent cancer cells, amongst other factors.

\section{Author Statement}

Research funding: this research has been developed partially with funding from Forschungszentrum Medizintechnik Hamburg (FMTHH) through project grant 02fmthh2018. Mrs. Viviane Teixeira thanks ProExzellenzia 4.0 program for providing the scholarship to continue her research. Conflict of interest: Authors state no conflict of interest. Ethical approval: animal experiments was approved by the local committee (Behörde für Gesundheit und Verbraucherschutz, Amt für Verbraucherschutz, Lebensmittelsicherheit und Veterinärwesen, assigned project No. N08/2018), supervised by the institutional animal welfare officer and performed in accordance with relevant guidelines and regulations.

\section{References}

[1] V.S. Teixeira, W. Krautschneider, J.J. Montero-Rodriguez, Bioimpedance Spectroscopy for Characterization of Healthy and Cancerous Tissues, in: 2018 IEEE Int Conf on Electrical Eng Phot (EExPolytech), IEEE, pp. 147-151.

[2] B. Tunstall, et. al, An analysis of systematic errors in the electrical impedance measurement of tissue characteristics during an in-vitro study in the frequency range $1 \mathrm{kHz}$ to $4 \mathrm{MHz}$, in: Proc 19th An Int Conf IEEE Eng in Med and Biol Soc., IEEE, 30 Oct.-2 Nov. 1997, pp. 860-862.

[3] A.J. Surowiec, et. al. Dielectric properties of breast carcinoma and the surrounding tissues, IEEE transactions on bio-medical engineering 35 (1988) 257-263.

[4] A.P. O'Rourke, et. al. Dielectric properties of human normal, malignant and cirrhotic liver tissue: in vivo and ex vivo measurements from 0.5 to $20 \mathrm{GHz}$ using a precision openended coaxial probe, Ph med biol 52 (2007) 4707-4719.

[5] J. Jossinet, M. Schmitt, A review of parameters for the bioelectrical characterization of breast tissue, Annals of the New York Academy of Sciences 873 (1999) 30-41.

[6] R.J. Halter, et. al. Electrical impedance spectroscopy of benign and malignant prostatic tissues, J of Ur 179 (2008).

[7] D. Haemmerich, et. al, In vivo electrical conductivity of hepatic tumours, Phys meas 24 (2003) 251-260.

[8] Q. Guofeng, et. al, Bioimpedance analysis for the characterization of breast cancer cells in suspension, IEEE trans on bio-medical engineering 59 (2012) 2321-2329.

[9] V.S. Teixeira, et. al. Electrical Impedance Spectroscopy for Characterization of Prostate PC-3 and DU 145 Cancer Cells, Int Conf IEEE Eng Med Biol Soc (EMBC). (2019) 6485-6489.

[10] J. Stachura, et. al. The dual role of tumor lymphatic vessels in dissemination of metastases and immune response development, Oncoimmunology 5 (2016) e1182278.

[11] A.I. Minchinton, I.F. Tannock, Drug penetration in solid tumours, Nature reviews. Cancer 6 (2006) 583-592.

[12] A.W. Lund, M.A. Swartz, Role of lymphatic vessels in tumor immunity: passive conduits or active participants?, Journal of mammary gland biology and neoplasia 15 (2010) 341-352.

[13] T.R. Samatov, V.V. Galatenko, A. Block, M.Y. Shkurnikov, A.G. Tonevitsky, U. Schumacher, Novel biomarkers in cancer: The whole is greater than the sum of its parts, Seminars in cancer biology 45 (2017) 50-57.

[14] C.L. River, The CB17/lcr-Prkdc-scid//crlcoCrl Mouse: A Fox Chase SCID (Severe Combined Immunodeficiecy) Model: The congenic CB17 SCID mouse model is commonly used in tumor biology, xenograft research, transplantation and immunology studies. 